\title{
The material-weight illusion induced by expectations alone
}

\author{
Gavin Buckingham • Nathalie S. Ranger • \\ Melvyn A. Goodale
}

Published online: 9 November 2010

(C) Psychonomic Society, Inc. 2010

\begin{abstract}
In the material-weight illusion (MWI), equally weighted objects that appear to be made from different materials are incorrectly perceived as having different weights when they are lifted one after the other. Here, we show that continuous visual experience of the lift is not a prerequisite for this compelling misperception of weight; merely priming the lifters' expectations of heaviness is sufficient for them to experience a robust MWI. Furthermore, these expectations continued to influence the load force used to lift MWIinducing stimuli trial after trial, supporting the notion that vision plays an important role in the skillful lifting of objects.
\end{abstract}

Keywords Weight illusions · Fingertip force scaling ·

Object lifting $\cdot$ Vision

Weight illusions provide a powerful demonstration of the subjectivity of our perception of heaviness. In the sizeweight illusion (SWI), similar-looking objects of different sizes but identical mass feel as if they weigh different amounts (Charpentier, 1891). Smaller objects feel substantially heavier than larger objects - an illusory difference in weight that does not diminish with repeated lifts or with the knowledge that the stimuli have identical mass (Flanagan \& Beltzner, 2000). Similarly, the material-weight illusion (MWI) can be induced by varying the material properties, rather than the size, of the stimulus set (Ellis \& Lederman, 1999; Harshfield \& DeHardt, 1970; Seashore, 1899; Wolfe, 1898). For example, adding a core of lead to increase the mass of a cube of polystyrene to match that of an

G. Buckingham $(\bowtie) \cdot$ N. S. Ranger $\cdot$ M. A. Goodale

Centre for Brain and Mind, University of Western Ontario,

London, ON, Canada

e-mail: gbucking@uwo.ca identically sized cube of metal will result in the compelling percept that the polystyrene outweighs the metal.

For over 100 years, researchers have struggled to understand what causes these illusions. One promising line of research has suggested that these illusions are caused by mismatches between the expected dynamics of the lift (efference) and the sensory consequences of the lift (afference). Individuals apply too much force to items that they imagined are heavy (i.e., the larger of the SWI stimuli) and too little force to items that they imagined are light. And it is these mismatches between motor prediction and feedback that result in the illusory percept. This compelling suggestion, however, does not tally well with the forces that individuals use to lift illusion-inducing objects repeatedly. Rather than persisting with the errors in force application that would underlie the sensorimotor mismatch, participants rapidly scale their motor plans to the actual (and identical) mass of the illusion-inducing objects. And, in spite of this rapid force scaling, the magnitudes of the SWI and MWI are not diminished (Buckingham, Cant, \& Goodale, 2009; Flanagan \& Beltzner, 2000; Grandy \& Westwood, 2006). The stark independence of perception and action in these tasks argues against a sensorimotor cause for weight illusions.

Recently, researchers have suggested that these illusions must have cognitive underpinnings. In this framework, individuals have long-held, experience-based priors about how heavy various objects will be, based on their perceptual properties. Large items are expected to be heavier than small items, and blocks of metal are expected to outweigh blocks of Styrofoam, reflecting our repeated experiences of the statistical properties of the environment (Flanagan, Bittner, \& Johansson, 2008). Crucially, however, these priors are very wide-ranging, contrasting the extremely flexible single-object motor 
plans that are utilized to lift individual objects, and are difficult to alter. And it is because of the stability of these cognitive priors that we perceive weight illusions long after our motor system has altered the way in which we interact with the stimuli.

A particularly crucial component for this representationbased theory of weight illusions is the intuitive idea that expectations are a persistent phenomenon. Continuous, direct perception of the various illusion-inducing properties (size/material) for the entire lift should not be required in order to induce the relevant expectations of heaviness. Instead, the knowledge alone that you will be lifting something of a certain weight, irrespective of how or when it is obtained, should be sufficient to drive the illusion. In a recent study (Buckingham \& Goodale, 2010), we showed that this was indeed the case with the SWI. Participants received a brief visual preview of a small, medium, or large cube that they thought they would eventually lift, before having their vision removed for the remainder of the trial. The previewed cube was then discreetly replaced with the medium-sized cube - the only item that participants lifted during the experiment. Despite the fact that participants lifted the same cube over and over again, they reported that it weighed different amounts as a function of what they had seen beforehand; it felt heavier after participants had seen the small cube than it did after they had seen the large cube. In other words, the SWI in this study was induced purely by expectations of heaviness, and these expectations persisted long after the vision of the cube had been removed. This finding was in stark contrast to that of earlier work suggesting that continuous experience of the object's size (through vision or haptics) was necessary to experience the SWI (Masin \& Crestoni, 1988).

Although our earlier SWI study (Buckingham \& Goodale, 2010) provided strong support for a representation-based theory of the SWI, it was unclear whether these representations extended beyond visual size into other, more variable visual indicators of mass, such as surface material. For example, the way in which we associate a particular material with a weight may operate on a less fundamental level than do size-based representations, given how variable such associations may be (a metal container filled with a less dense material is not an uncommon occurrence). If this is the case, the MWI may be a result of conflicting visual and proprioceptive sensory inputs, rather than of expectations of heaviness. Alternatively, our cognitive expectations of heaviness may be so wide-ranging that, regardless of how they are elicited, they underpin our perception of an object's weight. In the present study, we hoped to determine whether our cognitive representations of object weight are derived from their material properties, in the same way that we have shown them to be with object size (Buckingham \& Goodale, 2010). If expectations are critical for judging the weights of objects, irrespective of the particular context, we would expect the MWI to be readily experienced without the participants' viewing the lift itself. To test this hypothesis, we asked participants to lift identically weighted cubes that appeared to be made from different materials while wearing shutter goggles that obscured their vision. Before the lift, participants were given a short preview period in which they were allowed to see the cube they eventually would lift, priming their expectations of heaviness. If the block felt as if had a different weight (i.e., if participants experienced the MWI), we would be able to make stronger and more generalized conclusions regarding the extent to which our perception of heaviness is grounded in our cognitive expectations. A secondary purpose was to extend our preliminary investigation of fingertip force scaling in these illusions in the absence of vision. In our SWI data (Buckingham \& Goodale, 2010), we noted that participants failed to detect and correct their initial expectationbased errors, continuing to erroneously apply higher rates of force to the larger block than to the smaller block for a far longer time than they did when vision was permitted. Given the conceptual similarity between the SWI and MWI, we hoped to investigate whether participants would have equivalent difficulties with scaling their fingertip forces to each cube's actual mass.

\section{Method}

Twenty-five right-handed undergraduates from the University of Western Ontario participated in this study. Three participants were removed due to unusual lifting dynamics (multiple lifts with force rates greater than 2 standard deviations from the mean), leaving a sample of 22 (5 male, 17 female; mean age $=20.5$ years, $S D=7.3$ ). Participants, who were unaware of the existence of the MWI, gave written informed consent prior to testing, with all procedures approved by the University of Western Ontario research ethics board.

Participants sat in front of a table wearing closed PLATO shutter goggles. The experimenter attached a plastic handle containing a pair of force transducers to one of the MWI stimuli and placed it on a liftoff pad situated on the tabletop. The MWI stimuli were all $10 \times$ $10 \times 10 \mathrm{~cm}$ cubes. One was made from expanded polystyrene, another from oak, and one from aluminum. All the cubes were adjusted so as to weigh $700 \mathrm{~g}$ (see Buckingham et al., 2009, for further details about the creation and appearance of the stimuli). Once the object was in front of the participants, the goggles opened, 
allowing the participants to see the object that they were going to lift. One second later, an auditory cue signaled to the participants that they should reach out, grasp the force transducer handle with their dominant hand's thumb and index finger, and lift the object vertically upwards. When a force exceeding $1 \mathrm{~N}$ was applied to the force transducer handle (i.e., upon contact, but prior to liftoff), the PLATO goggles were closed, eliminating visual feedback for the lift. This procedure meant that participants could view the cube that they would lift right up until the lift commenced. Participants were told to lift in a smooth, confident, controlled manner until the bottom surface of the cube was approximately $5 \mathrm{~cm}$ above the table surface and to hold the object steady, without hefting it. Four seconds after the cue to lift had been given, a second auditory tone indicated that participants could return the object to the tabletop, at which point they gave an unconstrained number representing the object's heaviness (i.e., an absolute magnitude estimation; Zwislocki \& Goodman, 1980). Participants lifted each cube 15 times in randomized triplets of all three material types, in one of three orders, for a total of 45 lifts in a single session lasting approximately $30 \mathrm{~min}$. With the exception of participants being unable to see themselves lift the objects, this procedure was identical (and directly comparable) to that in our earlier MWI study (Buckingham et al., 2009).

Perceptual ratings of heaviness were normalized to a $z$ score distribution in order to account for individual differences in the range of scores reported. Electrical signal data from the force transducer was recorded at $1000 \mathrm{~Hz}$ and was converted into force vectors. Grip force was defined as the sum of the finger and thumb forces perpendicular to the grasp handles (representing the squeezing force), and load force was defined as the sum of the thumb and finger forces parallel to the grasp handles (mainly representing the vertical lift force). These forces were dual-pass Butterworth filtered at $14 \mathrm{~Hz}$ and were differentiated with a 3-point central difference equation to yield their rates of change. In accordance with our previous studies, the maximum grip and load forces and the maximum grip and load force rates, in addition to the load phase duration (the time that elapsed between the onset of loading force and the object's leaving the table, signaled by the liftoff pad), were used as the kinetic dependent variables. The perceptual and kinetic measures were examined in separate 3 (material) $\times 15$ (trial) ANOVAs with repeated measures. To determine whether any deficits in scaling were present when vision was removed, we compared the average difference between the forces applied to the metal and polystyrene cubes across the visual conditions with a one-way (between-subjects) ANOVA. Finally, Tukey tests were used to compare the forces used to lift the metal and polystyrene cubes on each of the first five trials in order to provide an indication of the rapidity and quality of the fingertip force scaling.

\section{Results}

Overall, the identically weighted cubes made from different materials were reported as feeling as if they weighed different amounts [main effect of material, $F(2,42)=5.37$, $p<.01]]$. Thus, participants judged the polystyrene cube (average normalized rating: 0.19) to weigh more than both the wood (average normalized rating: -0.06) and metal (average normalized rating: -0.13 ) cubes. No material $\times$ lift interaction was observed $(p=.07)$. These findings, combined with the linear trends in Fig. $1 \mathrm{~b}$, indicate that the magnitude of the illusion did not substantially change with repeated lifts. In addition, a main effect of trial was observed, $F(14,294)=20.31, p<.001$, indicating that all the blocks felt heavier as the experiment progressed. These data (Fig. 1b), although different in magnitude, are directionally very similar to those for our earlier full-vision MWI (Fig. 1a; reprinted from Buckingham et al., 2009).

Upon first inspection, the kinetic data appear largely consistent with those in Buckingham et al. (2009). As in our previous full-vision work, both the maximum grip and load forces showed main effects of material [grip force, $F(2,42)=4.66, p<.05$; load force, $F(2,42)=13.15$, $p<.001]$, without the material $\times$ trial interaction that generally characterizes rapid scaling of the fingertip forces (grip force, $p=.69$; load force, $p=.10$; Fig. 1d, f). These main effects indicate an overall tendency to apply more grip and load force to the metal cube than to the polystyrene one over the course of the entire experiment. However, these comparisons alone do not provide an adequate overview of the differences between the studies/visual conditions; it is clear from Fig. 1e, f that there are substantial differences between the loading forces applied to each of the cubes with and without vision. To better describe these differences between full vision and no vision, we first compared the average difference scores between the load forces used to lift the metal and polystyrene cubes (i.e., the effect that the material had on the motor system throughout the entire experiment). This analysis provided some tentative indications that individuals may have difficulty scaling their load forces without vision (i.e., a trend toward a larger difference score without vision than with vision) $F(1,42)=3.51$, $p=.07$. To examine the force scaling in a focused way, we then compared the differences between the load forces used to lift the large and small blocks over the first five trials, where any differences in scaling would be most pronounced. In our full-vision data set (Fig. 1e; reprinted from Buckingham et al., 2009), participants applied relatively similar loadings to all three cubes - so much so that none of 


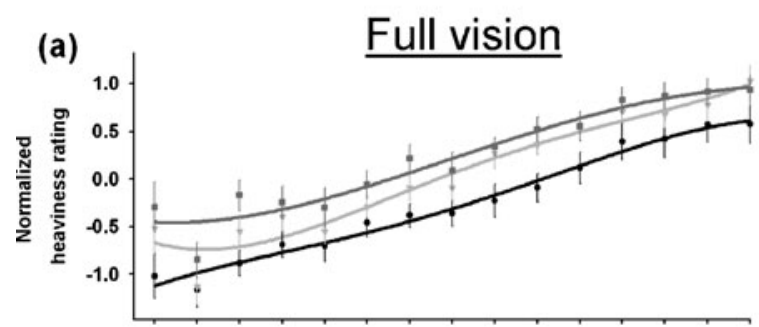

(b)
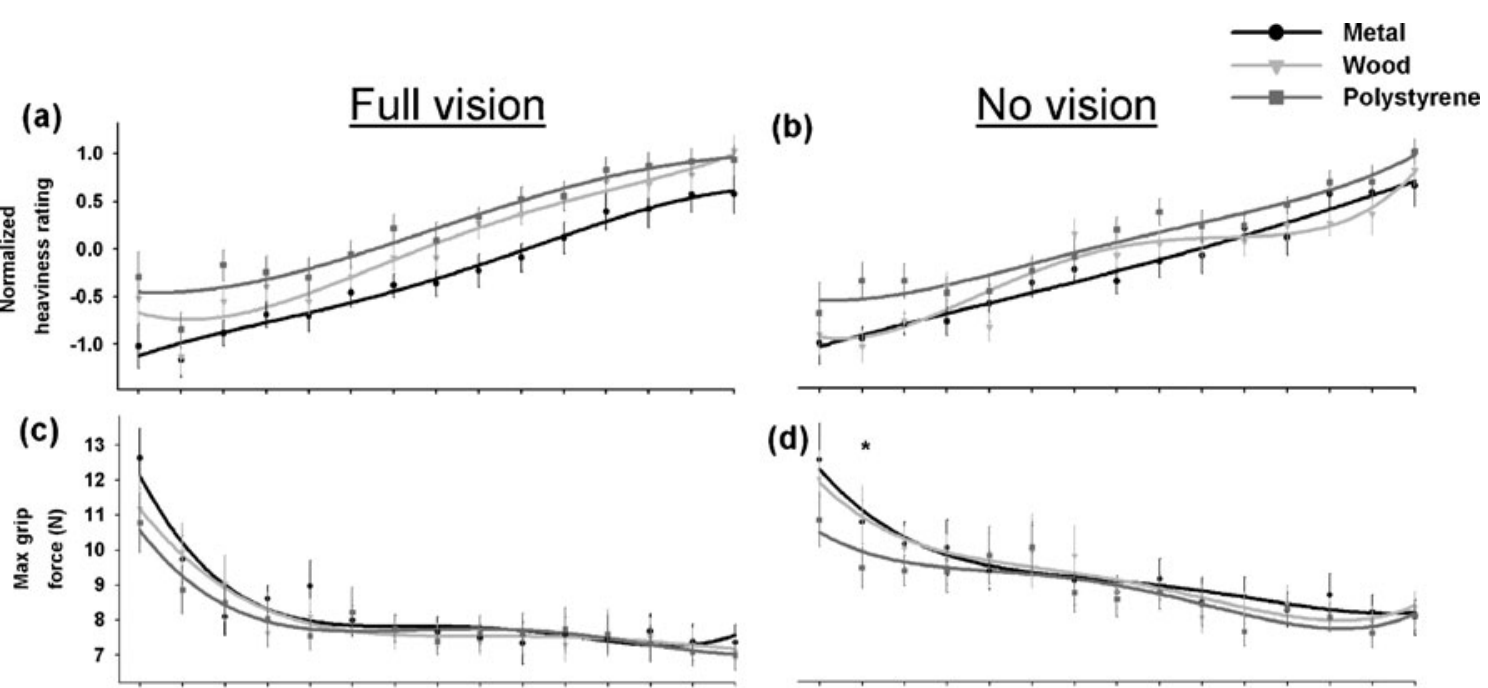

(d)!

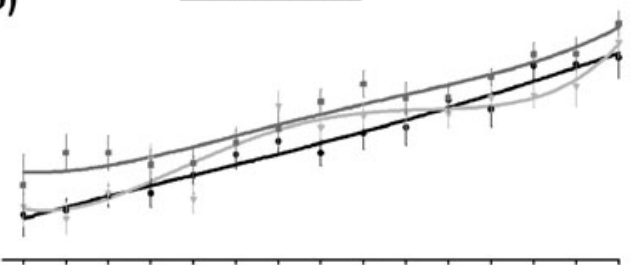

(e)
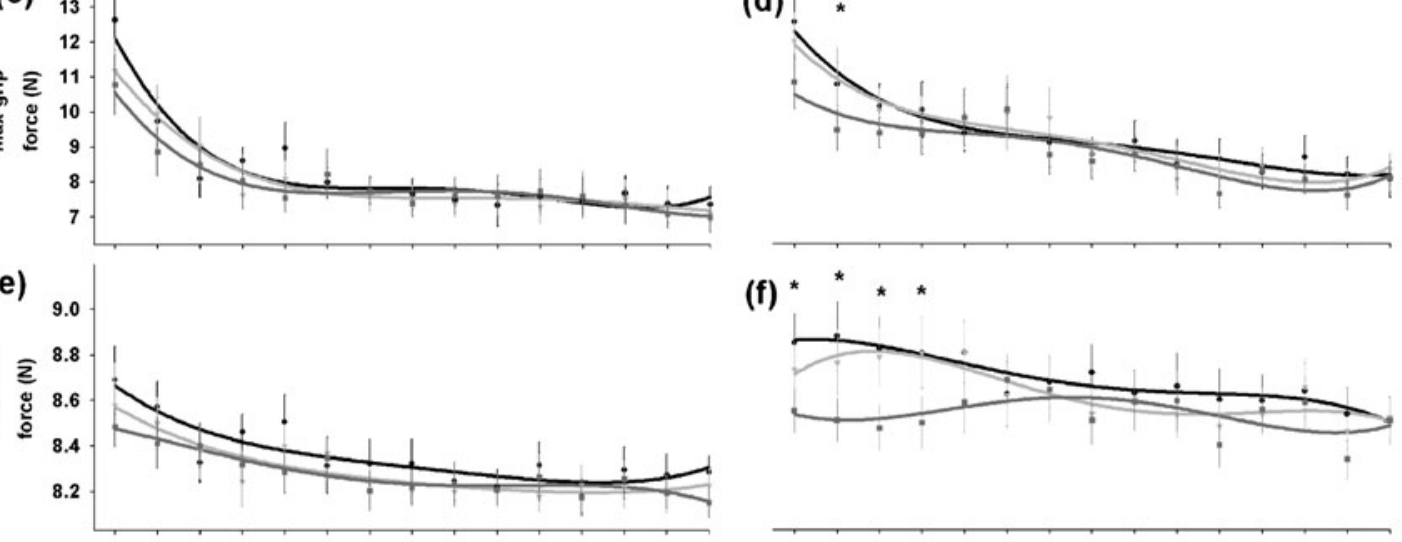

(f) *

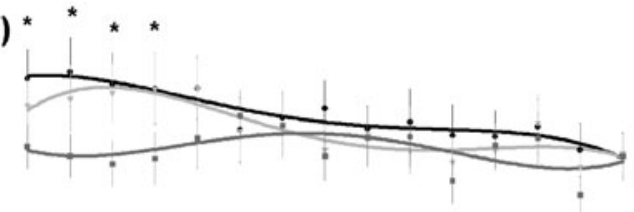

(g)

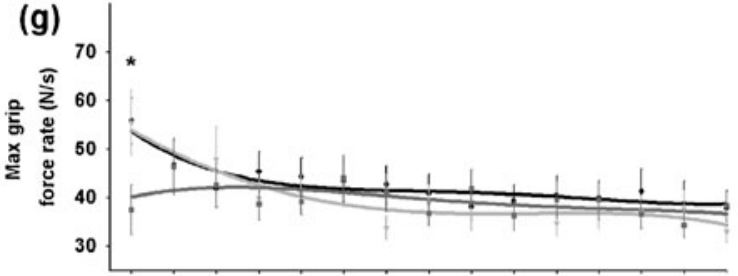

(h)
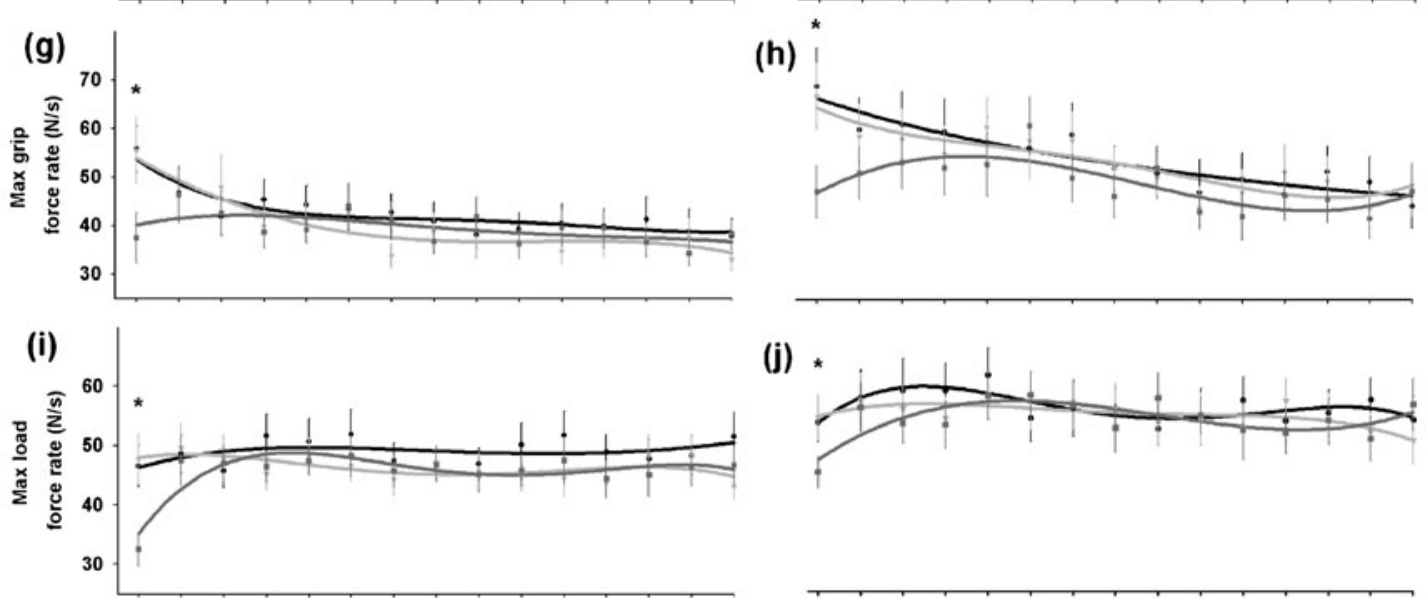

(j) *
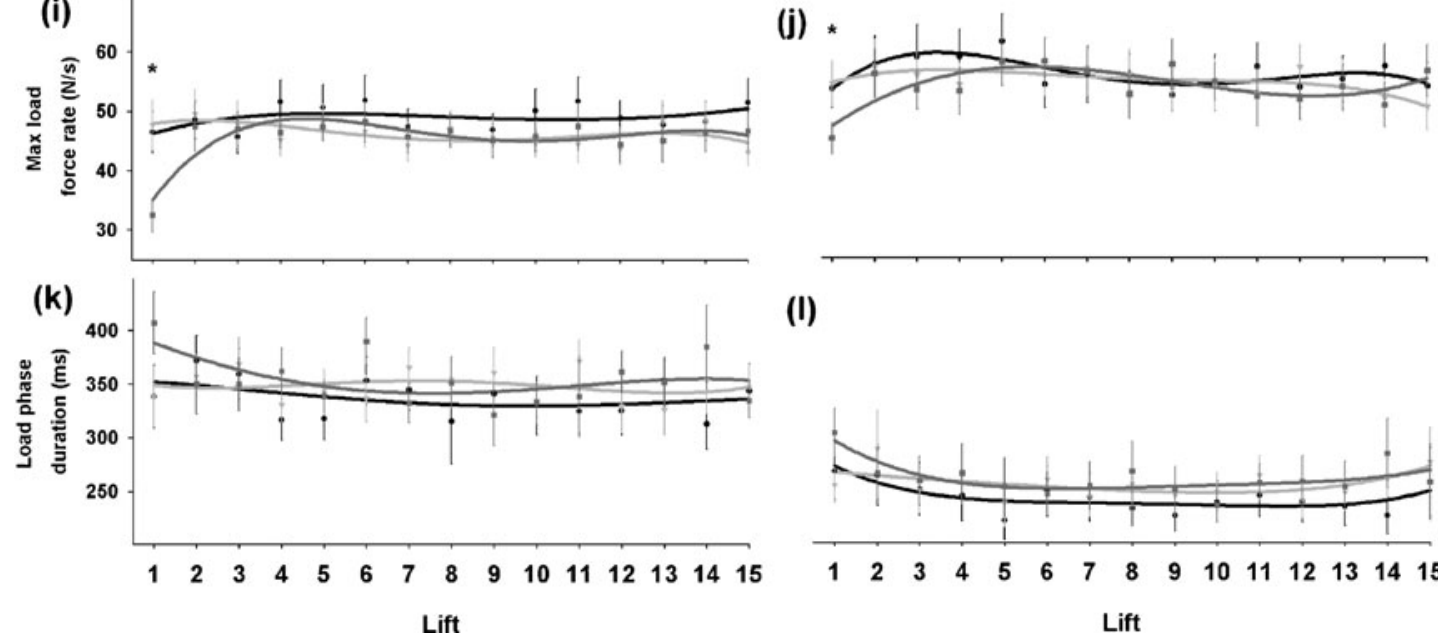

(I)

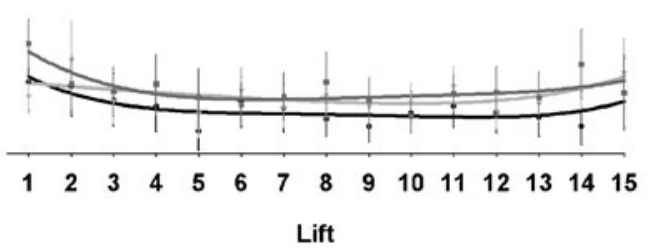

Fig. 1 Comparisons of the perceptual ratings (a, b) and kinetic measures (c-l) while MWI cubes were lifted with and without vision over all lifts. The data are fitted with fourth-order polynomials to visually identify the mean trends. Error bars represent between-

subjects standard errors of the means. The full-vision graphs (left column) are modified from Buckingham, Cant, and Goodale, 2009; Journal of Neurophysiology (2009); Am Physiol Soc, with permission 
the Tukey tests between the metal and polystyrene blocks on the first five trials yielded a significant difference. However, applying the same five Tukey tests as those described above to the present, no-vision data set yielded four significant differences. This means that, several times after experiencing its actual weight, participants continued to lift the metal cube with a greater load force (i.e., a greater acceleration) than they used to lift the polystyrene cube. This finding is consistent with our previous SWI work (Buckingham \& Goodale, 2010), where removing vision affected individuals' abilities to scale their fingertip forces accurately to each cube's actual mass.

In our previous SWI work, we noted that the largest effects of vision were on the force rates, rather than on the (unreported) forces themselves (Buckingham \& Goodale, 2010). Here, however, there was no such deficiency in the scaling of the grip and load force rates. A main effect of material was observed for maximum grip force rate, $F(2,42)=8.32, p<.005$, but not for the maximum load force rate, $p=.19$ ). Trial $\times$ material interactions were not present for either measure (grip force rate, $p=.18$; load force rate, $p=.30$ ). Likewise, load phase duration yielded a main effect of material, $F(2,42)=10.79, p<.001$, but no trial $\times$ material interaction, $p=.59$. It is clear from Fig. $1 \mathrm{~g}-1$ that, in all of the latter measures, there were no substantial differences across study/visual condition.

\section{Discussion}

In the present study, we examined whether or not expectations alone were sufficient to induce an MWI, even without any visual experience of lifting. As we have argued elsewhere (Buckingham \& Goodale, 2010), it is possible that the integration of vision and proprioception during a lift plays a causal role in the illusion, and it is thus important to eliminate this possibility when evaluating how expectations alone contribute to the MWI and SWI. Participants viewed cubes that appeared to be made from metal, wood, and polystyrene, which had been adjusted to weigh the same, before lifting them without vision. Despite having no continuous percept of each cube's material (see Ellis \& Lederman, 1999), participants still experienced a robust MWI:The metal cube was reported as feeling lighter than the polystyrene one. Interestingly, the magnitude of the illusion (average metal cube rating subtracted from the average polystyrene cube rating) in the present work is $57 \%$ smaller than the one reported in our prior full-vision MWI experiment (Buckingham et al., 2009). This reduction in illusion strength was also noted in our recent SWI work, where removing vision made the SWI 51\% smaller (Buckingham \& Goodale, 2010). Furthermore, the multiple factors that were varied between the vision and no-vision conditions in our previous SWI task (e.g., that participants lifted only a single block and lifted after a substantial delay from the visual preview) are not relevant in the present MWI work. In other words, our full-vision and no-vision tasks are directly comparable with one another. The results of the present experiment therefore make it clear that continuous visual experience is not a prerequisite for experiencing any sort of weight illusion. Instead, viewing the lifting hand appears to modulate the strength of an illusion that has already been set in place by cognitive expectations of weight. Thus, by removing vision of the lifts, we have isolated the (evidently substantial) cognitive contribution that expectations make to the illusory experience. Clearly, the weight that a lifter cognitively expects an object to be has a large impact on how heavy it eventually feels when it is lifted. Furthermore, it is not simply the size of an object that is represented strongly enough to influence perception of weight, but also the higher-level learned associations of visual material properties. The expectations of heaviness not only affected how heavy each cube felt, but also impacted the way in which the cubes were lifted. During their initial interactions with the stimuli, participants applied greater forces and higher force rates to the metal cube than they did to the polystyrene cube. This pattern of behavior is similar to what occurs during full-vision MWI lifts (Buckingham et al., 2009). When individuals are permitted to watch themselves lift these stimuli, however, a very rapid scaling of the fingertip forces and force rates occurs; seeing and feeling oneself lift MWI stimuli a single time is enough to detect and correct the expectation-based errors. Without vision, however, participants appeared to have some difficulty scaling their load forces. A naïve prediction for repeated lifts without vision may have been that participants would start applying forces in line with their perceptual experience of heaviness-applying more force to the heavier feeling polystyrene cube. Instead, participants persisted in applying load forces in line with their expectations of heaviness, with our sample taking at least four lifts of each of the cubes to correct their errors. This finding highlights the counterintuitive and generally overlooked role that vision must play in the detection and correction of ostensibly haptic lift errors (Buckingham \& Goodale, 2010). It is worth noting that vision's impact on fingertip force scaling appears to differ between the SWI and MWI. In the SWI, removing vision impaired scaling in the force rates (Buckingham \& Goodale, 2010), whereas in the MWI, the same manipulation impaired the scaling of only the maximum load force. We propose that this discrepancy highlights differences in the way that the motor system reacts to the factors that cause the illusions - object size and object material, respectively. In contrast to the (apparently very similar) cognitive representations that underlie the perceptual illusions, the motor system 
treats size- and material-induced expectations differently. In other words, repeated lifts without vision are more likely to be influenced by an object's size, rather than by its material. We suspect that the motor system treats material and size cues differently because of the relative frequency of these associations within the environment: The relationship between visual size and weight is more constant than the relationship between the apparent material and weight. Thus, the perceptual system's use of general expectations of heaviness does not extend to the motor system, which relies more on cues for size than on those for material. Here, we have demonstrated that, much like the SWI, the MWI can be induced without vision. Furthermore, load forces were driven by these expectations of heaviness in the direction opposite to the illusory weight difference, and these expectation-based errors persisted long after they would have been corrected had vision of the lift been allowed. In sum, the present work provides a powerful demonstration of how cognitive expectations can, in isolation, impact our perception of heaviness and the way in which we interact with the world around us.

\footnotetext{
Acknowledgements We would like to thank J. Ladich for constructing the MWI stimuli, in addition to H. Yang for technical support. This research was funded by a Canadian Institutes of Health Research (CIHR) operating grant awarded to M. A. Goodale, and a Natural Sciences and Engineering Research Council of Canada (NSERC) summer studentship awarded to N. Ranger.
}

\section{References}

Buckingham, G., Cant, J. S., \& Goodale, M. A. (2009). Living in a material world: How visual cues to material properties affect the way that we lift objects and perceive their weight. Journal of Neurophysiology, 102, 3111-3118.

Buckingham, G., \& Goodale, M. A. (2010). Lifting without seeing: the role of vision in perceiving and acting upon the size weight illusion. PLOS ONE, 5, e9709. doi:10.1371/journal.pone.0009709

Charpentier, A. (1891). Analyse expérimentale: De quelque éléments de la sensation de poids. Archives de Physiologie Normale et Pathologique, 3, 122-135.

Ellis, R. R., \& Lederman, S. J. (1999). The material-weight illusion revisited. Perception \& Psychophysics, 61, 1564-1576.

Flanagan, J. R., \& Beltzner, M. A. (2000). Independence of perceptual and sensorimotor predictions in the size-weight illusion. Nature Neuroscience, 3, 737-741.

Flanagan, J. R., Bittner, J. P., \& Johansson, R. S. (2008). Experience can change distinct size-weight priors engaged when lifting objects and judging their weights. Current Biology, 18, 1742-1747.

Grandy, M. S., \& Westwood, D. A. (2006). Opposite perceptual and sensorimotor responses to a size-weight illusion. Journal of Neurophysiology, 95, 3887-3892.

Harshfield, S. P., \& DeHardt, D. C. (1970). Weight judgment as a function of apparent density of objects. Psychonomic Science, 20, 365-366.

Masin, C. M., \& Crestoni, L. (1988). Experimental demonstration of the sensory basis of the size-weight illusion. Perception \& Psychophysics, 44, 309-312.

Seashore, C. E. (1899). Some psychological statistics: II. The material weight illusion. University of Iowa Studies in Psychology, 2, 36-46.

Wolfe, H. K. (1898). Some effects of size on judgments of weight. Psychological Review, 5, 25-54.

Zwislocki, J. J., \& Goodman, D. A. (1980). Absolute scaling of sensory magnitudes: A validation. Perception \& Psychophysics, 28, 28-38. 